\title{
Joint spatial-frequency distribution of Gaussian signals and prospects of its applications for description of optical systems
}

\author{
Kozlovskii Yu. M. \\ Institute for Condensed Matter Physics, 1 Sventsitski, 79011 Lviv, Ukraine, \\ e-mail: nesh@icmp.lviv.ua
}

Received: 17.01.2008

In final form: 01.04.2008

\begin{abstract}
We consider a joint spatial-frequency distribution as a result of superposition of two known Wigner and Ville distributions. The joint distribution is characterized by a certain parameter $t$ that plays a part of measure of superposition: the joint frequency-spatial distribution passes into the Ville one at $t=0$ and coincides with the Wigner distribution at $t=1$. For the values $0<t<1$ there exists a row of 'mixed' distributions, which represent superposition of the Wigner and Ville distributions. Explicit expression for the joint distribution of Gaussian signal is obtained by means of analytical calculations. We present graphic illustrations for the shape of joint distribution of Gaussian signal at different values of the joint parameter $t$. It is demonstrated that the Wigner distribution is formed as a rotation of Ville distribution on the information diagram by the angle proportional to the joint parameter $t$.
\end{abstract}

Keywords: time-frequency analysis, Wigner distribution, Ville distribution, Wigner tomography, Fourier optics

PACS: 05.60.+w, 05.70.Ln, 05.20.Dd, 52.25.Dg, 52.25.Fi

UDC: 535.8

\section{Introduction}

Nowadays different types of spatial-frequency distributions are successfully employed for analysis of nonstationary signals [1-2]. There is reliable information on both advantages and deficiencies of many of those distributions if they are applied in different fields of physics. Wigner and Ville ${ }^{1}$ distributions have found wide applications in spatial-frequency analysis, particularly in the optical systems for information processing. It is well known that just these distributions reveal some properties successfully used for

\footnotetext{
${ }^{1}$ In a number of works the term "Ville distribution" is referred to as "ambiguity function". Here we use the former term because the corresponding distribution has been introduced in the work by J.Ville, Cables et transmissions 2A, 61 (1948).
} 
description of various optical systems. Applications of distributions mentioned above are manifold and concern, in particular, theory of optical lens systems, theory of communications, acoustics, hydrolocation etc. [3-5]. Recent investigations have proven efficiency of the applications of spatial-frequency distributions in biology and medicine. For instance, it has been reported that the Wigner distribution could be applied for reconstructing 3D structures in frame of optical tomography [6-8].

One of the promising investigation directions within the spatial-frequency processing of signals is studying the properties of novel spatial-frequency representations of the distributions, with the aim of their further applications in different areas of physics and medicine. Unfortunately, it often happens that some spatial-frequency distributions do not meet demands raised by one or another specific application. In this relation, many of the existing distributions need generalization or improvements when applied to a given problem.

During the second half of the past century and the beginning of this one, a clear tendency has been observed towards generalization of different spatial-frequency distributions. The first attempt of such a generalization has been due to L. Cohen [9] as long ago as in 1966. The author has introduced a number of quasi-probability distributions that yield in correct quantum-mechanical marginal distributions. The Wigner distribution has been addressed there as an individual case. The next step has been done by N. De Bruijn in 1973 [10]. He has devoted his work to elaborating theory of generalized functions, with applications concerned with the Wigner and Ville distributions.

Summarizing the results of numerous studies, Cohen [1, 4] has suggested a generalized distribution involving a certain kernel dependent upon two parameters:

$$
\begin{aligned}
C(x ; \omega, \Phi) & =\frac{1}{2 \pi} \int f\left(y+\frac{x_{0}}{2}\right) f^{*}\left(y-\frac{x_{0}}{2}\right) \Phi(x, \omega) \\
& \times \exp \left(-i\left[\omega_{0} x-\omega_{0} x+\omega_{0} y\right]\right) d y d x_{0} d \omega_{0} .
\end{aligned}
$$

Depending on the form of the kernel $\Phi(x, \omega)$, this distribution degenerates into one of the known distributions (Wigner, Ville, Woodward, Kirkwood, Page, Mark, etc.). In general, Eq. (1) describes a class of spatial-frequency distributions, which has been later called as a Cohen's class. The distributions which are already known or still unknown can belong to the above class. Of course, the latter ones should necessarily satisfy all of the relevant requirements. In his studies, Cohen has given much attention to the problems of physical interpretation of different distributions, together with methodology for signal synthesis on the basis of appropriate distributions. The well-known specialist in the signal theory, A. Mertins, has also touched upon the theory of generalization of spatialfrequency distributions. In his monograph "Signal Analysis" [5] he has singled out this topic into a separate section "General spatial-frequency distributions". The author has stated that the Wigner distribution serves as an excellent tool for spatial-frequency 
analysis as long as a linear dependence is kept between the instantaneous coordinates and frequencies. Otherwise, a need in generalizing appears, whose general principles are described in detail in the mentioned work.

Among numerous recent studies related to generalizing spatial-frequency distributions, we should mention only the most typical ones. The PhD Thesis by L. Durak "Novel time-frequency analysis technique for deterministic signals" [11] is one of such studies, where a close attention has been paid to generalizing distributions and introducing their additional parameters.

Generalizations of spatial-frequency distributions have been thoroughly considered in the collection edited by B. Boashash [2]. Among a number of studies included in it, we would like to emphasize the works by R. Baraniuk (p. 123), X. Xia (p. 223) and A. Papandreou-Suppappola (p. 643). Within the mentioned collection, the work by G. Matz by F. Hlawatsch (p. 400) is of particular interest in relation to the problem of distributions generalization, since it considers methodology for constructing generalized distributions on the basis of both the Wigner distribution and the ambiguity function (i.e., the Ville distribution).

In the present work we try to use interlinks between the Wigner and Ville distributions with the aim of joining them into a single, more general distribution. Up to date, it has been revealed that the two distributions are related by a double Fourier transform. The results obtained by us allow tracing transformation of one of the distributions into the other, while changing the distribution parameter $t$. This generalized distribution generates a whole set of new distributions formed in the process of switching between the basic distributions. The latter fact may prove important from the viewpoint of possible practical applications.

Hitherto, a choice between the Ville and Wigner distributions remains ambiguous. Each of them has its own scheme for reconstruction of signal intensity distribution. So, the scheme adopted for the Wigner distribution includes calculating the marginal distributions [1]. The Ville distribution permits much simpler reconstruction scheme, owing to simpler mathematical transformations $[12,16]$. Traditionally, the Wigner distribution has been used in a large majority of studies performed within the field. Introduction of the joint distribution would mean a possibility for calculating 'mixed' states and determining the necessary contributions of each of the limiting distributions. Hence, there appears a possibility for revealing the state necessary for the system.

As mentioned before, there have been a number of techniques for generalizing distributions, which are concerned with different applied problems. However, only S. Chountasis has suggested the approach [13] that enables transitions between the Wigner and Ville distributions. Such the distributions play an important part in the analysis of phase space and, moreover, can be immediately applied in the Wigner tomography [6-8]. In 1999 Chountasis and co-authors [13-15] have developed a general distribution based on the Wigner formalism, which involves an additional parameter $\theta$. This study has been performed in frame of quantum-mechanical formalism. It allows 
passing the Wigner and Lille distributions into each other by means of changing the latter parameter.

The problem of calculation of a classical analogue of this generalized distribution remains urgent. It may be constructed based on the results [13] or using the formalism of Ville distribution, as has been done by the present author when studying the properties of fractal Fourier transform [17]. Similarly to the works [12, 16], the author has employed reconstruction of signal intensity based upon the Ville distribution. Meanwhile, it is just this reconstruction scheme that is realized experimentally in the real optical systems [17].

\section{Joint spatial-frequency distribution. Basic grounds}

In this work we propose to use a novel generalized distribution. The expression for this spatial-frequency distribution of signals $f_{1}(x)$ and $f_{2}(x)$ may be written as follows:

$$
\begin{aligned}
K_{f f^{*}}^{(t)}(x ; p) & =C_{t} \iint_{d x_{0}} d \omega_{0} \times \exp \left\{i\left[x_{0} p-\omega_{0} x\right]\right\} \exp \left\{-i \frac{\left(x-x_{0}\right)^{2}+\left(p-\omega_{0}\right)^{2}}{\tan (\theta / 2)}\right\} \times \\
& \times \int f_{1}\left(z+\frac{x_{0}}{2}\right) f_{2}^{*}\left(z-\frac{x_{0}}{2}\right) \exp \left(-i \omega_{0} z\right) d z .
\end{aligned}
$$

Here we adopt the notations

$$
C_{t}=\frac{2}{\pi} \frac{1}{1-\exp i \theta}, \quad t=\frac{\theta}{\pi},
$$

with $t$ being the parameter of the joint distribution and defining the form of representation. The limiting cases of Eq. (2) are the Ville distribution itself [9, 16] achieved at the value $t=0$,

$$
A_{f_{1} f_{2}^{*}}\left(x_{0} ; \omega_{0}\right)=\int f_{1}\left(x+\frac{x_{0}}{2}\right) f_{2}^{*}\left(x-\frac{x_{0}}{2}\right) \exp \left(-i \omega_{0} x\right) d x,
$$

and the Wigner distribution [1] reached at $t=1$,

$$
W_{f_{1} f_{2}^{*}}(x ; \omega)=\int f_{1}\left(x+\frac{x_{0}}{2}\right) f_{2}^{*}\left(x-\frac{x_{0}}{2}\right) \exp \left(-i \omega x_{0}\right) d x_{0} .
$$

The generalized distribution (2) may be represented in another form, which is often more convenient while performing mathematical calculations:

$$
\begin{aligned}
& K_{f_{1} f_{2}^{*}}^{(t)}(x ; p)=C_{t} \iint d x_{0} d \omega_{0} A_{f_{1} f_{2}^{*}}\left(x_{0} ; \omega_{0}\right) \times \\
& \exp \left\{i\left[x_{0} p-\omega_{0} x\right]\right\} \exp \left\{-i \frac{\left(x-x_{0}\right)^{2}+\left(p-\omega_{0}\right)^{2}}{\tan (\theta / 2)}\right\} .
\end{aligned}
$$

We call the generalized distribution introduced on the basis of Ville formalism as a joint spatial-frequency distribution of signals. A principled difference of the joint distribution (6) from the generalized Wigner function introduced by Chountasis [10] is that the exponent including the linear terms $x_{0}$ and $\omega_{0}$ does not contain the factor two. As a matter of fact, this difference, which might have been treated as insignificant, proves to be very essential in all the further calculations and reconstruction of signal after its 
distribution. The other difference is a presence of usual Ville distribution in the r. h. s. of Eq. (6). This approach allows reconstructing the intensity distribution for the input signal detected experimentally in the optical system. The scheme for reconstruction of the input signal following from the Ville distribution has been described in the works $[2,5,9,16]$. Thus, as an alternative to the known Wigner and Ville distributions, we would come to a set of 'mixed' distributions determined by some of the values $0<t<1$.

While working with the joint distribution, we deal in fact with a certain mixed state of our system, which should be described with the Wigner or Ville distributions in the limiting cases. It is known [2] that there are some problems whose solution makes one of those distributions more convenient, though the other problems do not enforce this choice and so the latter remains ambiguous. In the first place, this refers to the problems dealing with reconstruction of the input light intensity following from the known distribution. They are usually solved using the Wigner distribution $[1,2,4,5]$.

However, the studies $[12,16]$ have proven that the Ville distribution has some advantages in this respect. This is an example of problems of the type where the choice between the Wigner and Ville distributions is still ambiguous [2]. When employing the joint distribution and changing the joint parameter $t$, we can find the optimal state for the given system. This state can be described by the Wigner or Ville distribution or the joint distribution with some other parameter $t$. Considering universality of the problem mentioned, in this work we study the main properties of the suggested approach on the example of Gaussian signal.

\section{Joint spatial-frequency distribution of Gaussian signal}

Now let us pass to studying the main properties of the joint distribution and use, as an example, the Gaussian signal specified as

$$
g(x)=\frac{1}{2 \pi \sqrt{\sigma}} \exp \left(-\frac{1}{2 \sigma^{2}} x^{2}\right) .
$$

The Ville and Wigner distributions for the Gaussian signal are well known from the literature [5]. The corresponding Ville distribution has the form

$$
A_{g g^{\prime \prime}}\left(x_{0} ; \omega_{0}\right)=2 \sigma \exp \left(-\frac{1}{4}\left[\frac{x_{0}^{2}}{\sigma^{2}}+\omega_{0}^{2} \sigma^{2}\right]\right),
$$

while the Wigner distribution for the signal given by Eq. (7) is written as

$$
W_{g g^{\prime}}(x ; \omega)=\sigma \exp \left(-\left[\frac{x^{2}}{\sigma^{2}}+\omega^{2} \sigma^{2}\right]\right) .
$$

The distributions (8) and (9) differ both functionally and by the amplitude. Our task is to reveal mechanism for their mutual redistribution. For this aim we will calculate the joint distribution of the Gaussian signal. Inserting the Gaussian function (7) into definition of the joint distribution (2) and taking Eqs. (6) and (8) into consideration, we get

Ukr. J. Phys. Opt. 2008, V9, №2 


$$
K_{g g^{*}}^{(t)}(x ; p)=C_{t} \exp \left(-i \frac{p^{2}+x^{2}}{T}\right) I_{x_{0}}(x, p) I_{\omega_{0}}(x, p)
$$

where

$$
\begin{aligned}
& I_{x_{0}}(x, p)=\frac{\sqrt{\pi}}{\sqrt{a_{1}}} \exp \left\{-\frac{1}{a_{1}}\left(\frac{x}{T}+\frac{p}{2}\right)^{2}\right\}, \\
& I_{\omega_{0}}(x, p)=\frac{\sqrt{\pi}}{\sqrt{a_{2}}} \exp \left\{-\frac{1}{a_{2}}\left(\frac{p}{T}+\frac{x}{2}\right)^{2}\right\}, \\
& a_{1}=\frac{T+i 4 \sigma^{2}}{4 T \sigma^{2}}, \quad a_{2}=\frac{4 i+T \sigma^{2}}{4 T}, T=\tan \left(\frac{\theta}{2}\right) .
\end{aligned}
$$

After not too complicated mathematical transformations and substituting the expressions (11) and (12) into Eq. (13) one can obtain the following explicit analytical form of the joint distribution for the Gaussian signal:

$$
K_{g g^{*}}^{(t)}(x, p)=C_{t t} \exp \left\{-\frac{i}{T}\left(x^{2}+p^{2}\right)\right\} \times \exp \left\{-\frac{1}{a_{1}}\left(\frac{x}{T}+\frac{p}{2}\right)^{2}-\frac{1}{a_{2}}\left(\frac{p}{T}-\frac{x}{2}\right)^{2}\right\},
$$

where

$$
C_{t t}(\theta)=\frac{2}{1-\exp i \theta} \frac{\sigma}{\sqrt{a_{1} a_{2}}} .
$$

The appearance of the joint distribution for the Gaussian signal for different parameter values $0<t<1$ may be found by taking a necessary value of the joint parameter $t$ in Eq. (14).

\section{Limiting cases for the joint distribution of Gaussian signal}

Let us study the limiting cases of Eq. (14), when the parameter $T$ belongs to the domains of small and large values.

Case $t=1(T \rightarrow \infty)$

Consider the region of angles close to $\pi$, i.e. the values $\theta=\pi-\varphi$, where $\varphi<<1$. Then we have $\cos \theta=-\cos \varphi, \sin \theta=\sin \varphi$ and the constants dependent on the $\theta$ parameter become as follows:

$$
\lim _{T \rightarrow \infty} a_{1}=\frac{1}{4 \sigma^{2}}, \quad \lim _{T \rightarrow \infty} a_{2}=\frac{\sigma^{2}}{4}, \quad \lim _{T \rightarrow \infty} C_{t}=\frac{1}{\pi}\left(1+i \frac{\varphi}{2}\right), \quad \lim _{T \rightarrow \infty} C_{t t}(\theta)=4 \sigma .
$$

Therefore the joint distribution for the Gaussian signal for the limiting case $t=1$ may be rewritten as

$$
\lim _{T \rightarrow \infty} K_{g g^{*}}^{(t)}(x, p)=4 \sigma \exp \left(-\left[\frac{x^{2}}{\sigma^{2}}+p^{2} \sigma^{2}\right]\right) .
$$

Up to the factor two, Eq. (17) coincides with the Wigner distribution for the Gaussian signal (see Eq. (9)). The functional dependence of the joint distribution for the 
Gaussian signal on the coordinates $x$ and $p$, taken for the parameter value $t=1(\theta=\pi)$, coincides with the same dependence typical for the Wigner distribution.

Case $t=0(T \rightarrow 0)$

This case corresponds to the region of small $\theta$ angles. Let us mark that the coefficients $a_{1}$ and $a_{2}$ for this case become purely imaginary $\left(a_{1} \sim a_{2} \sim i / T\right)$. It is easily seen that the following equality holds true for the quantity $A=\left(a_{1} a_{2}\right)^{-1 / 2}$ :

$$
A=T\left[\frac{T^{2}}{8}\left(\sigma^{2}+\sigma^{-2}\right)-i\left(1-\frac{T^{2}}{64}\left(\sigma^{2}+\sigma^{-2}\right)\right)\right] \text {. }
$$

The constant value $C_{t}$ in the limit of $\theta \ll 1$ may be represented as

$$
C_{t}=\frac{1}{\pi}\left(1+\frac{i}{T}\right) \text {. }
$$

As a result, one can arrive at the expression

$$
A C_{t}=\frac{1}{\pi}(T+i)\left[\frac{T}{8}\left(\sigma^{2}+\sigma^{-2}\right)-i\right]
$$

for the product of Eqs. (18) and (19), which is equal to $\pi^{-1}$ in the limit $T \rightarrow 0$. After that one has to eliminate the uncertainties available in the exponent of the joint Gaussian distribution (14). One can prove that mathematical transformations of Eq. (14) yield in the following result:

$$
\begin{aligned}
& K_{g g^{*}}^{(t)}(x, p)=C_{t t} \exp \left\{-\left(\frac{x^{2}}{4 \sigma^{2}}+\frac{p^{2} \sigma^{2}}{4}\right)\right\} \times \\
& \times \exp \left\{-\frac{T p x}{4}\left(\sigma^{2}+\frac{1}{\sigma^{2}}\right)-\frac{T^{2} p^{2}}{16 \sigma^{2}}-\frac{T^{2} x^{2} \sigma^{2}}{16}\right\} \times \\
& \times \exp \left\{-\frac{i}{T}\left(x^{2}+p^{2}\right)\right\} .
\end{aligned}
$$

It is seen that Eq. (21) acquires the form

$$
\lim _{T \rightarrow 0} K_{g g^{\prime \prime}}^{(t)}(x, p)=\sigma \exp \left(-\frac{1}{4}\left[\frac{x^{2}}{\sigma^{2}}+p^{2} \sigma^{2}\right]\right)
$$

in the limiting case of $T \rightarrow 0$. Hence, the joint distribution for the Gaussian signal at the parameter $t=0(\theta=0)$ is equivalent to the Ville distribution.

\section{Calculation results for the joint distribution of Gaussian signal}

The analytical results obtained by us for the joint Gaussian distribution (see Eqs. (14), (17) and (22)) allow to illustrate visually the process of redistribution between the planes of Wigner and Ville distributions. Let us start our analysis with the Ville distribution and its transformation into the Wigner one, which is governed by changing the $t$ parameter from $t=0$ to $t=1$. The relevant results are depicted in Fig. 1 (for the real part) and Fig. 2 
(for the imaginary part). The case (a) corresponds to the joint parameter value of $t=0.001$ (i.e., the rotation angle of $\theta=0.18^{0}$ ).

Within the accuracy limits, the joint distribution formed at this $t$ value can be regarded as the Ville one. The real part of this distribution shown in Fig. 1a, d coincides with the Ville distribution calculated with the formula (8), whereas the magnitude of its imaginary part (see Fig. 2a, d) is vanishingly small (of the order of $10^{-4}$ ). Obviously, the
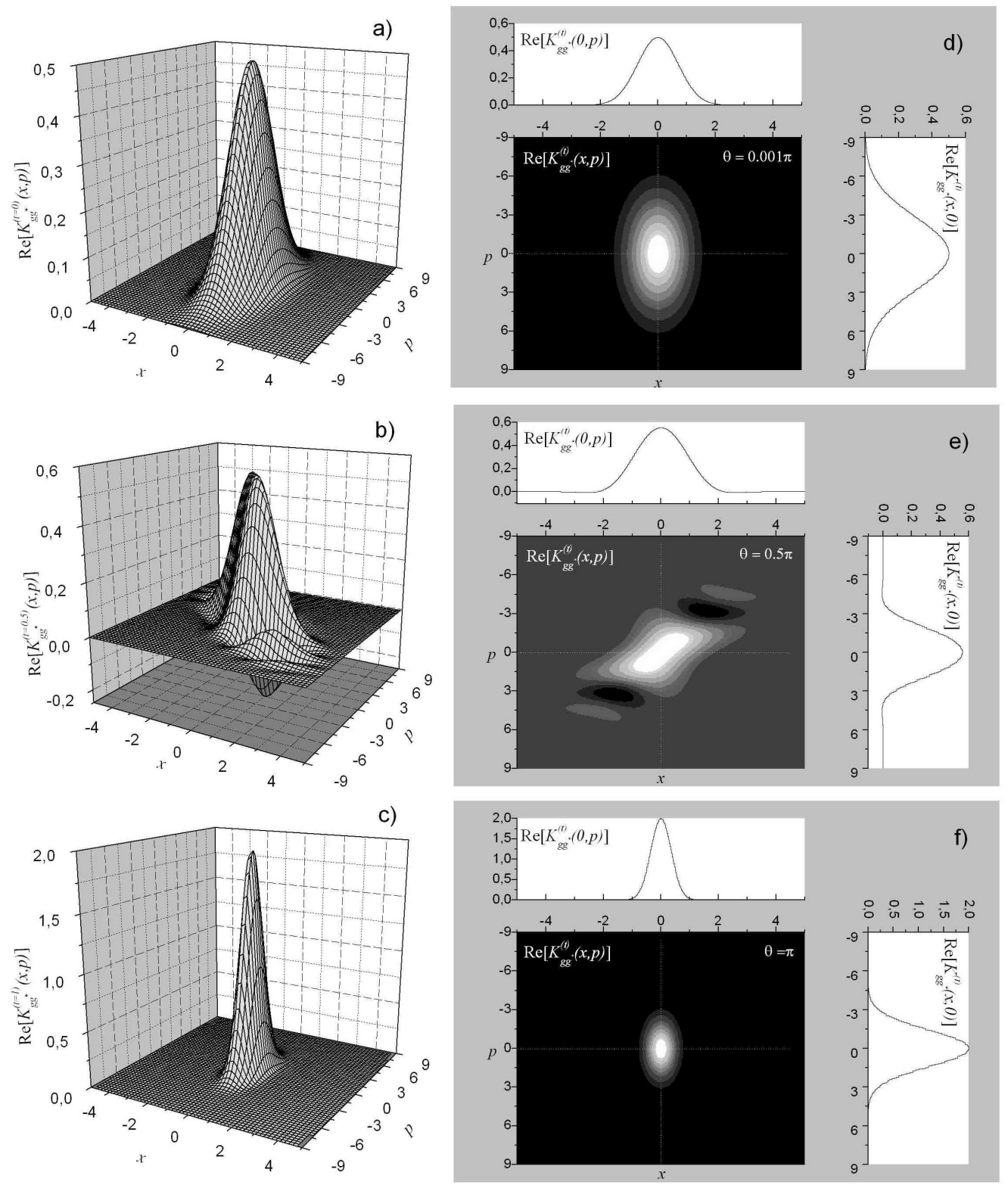

Fig. 1. Rotation of the joint distribution for the Gaussian signal (real part) on the information diagram at different values of the joint parameter and $\sigma=0,5: t=0.001$ (a, d), $t=0.5$ (b, e) and $t=1$ (c, f). 

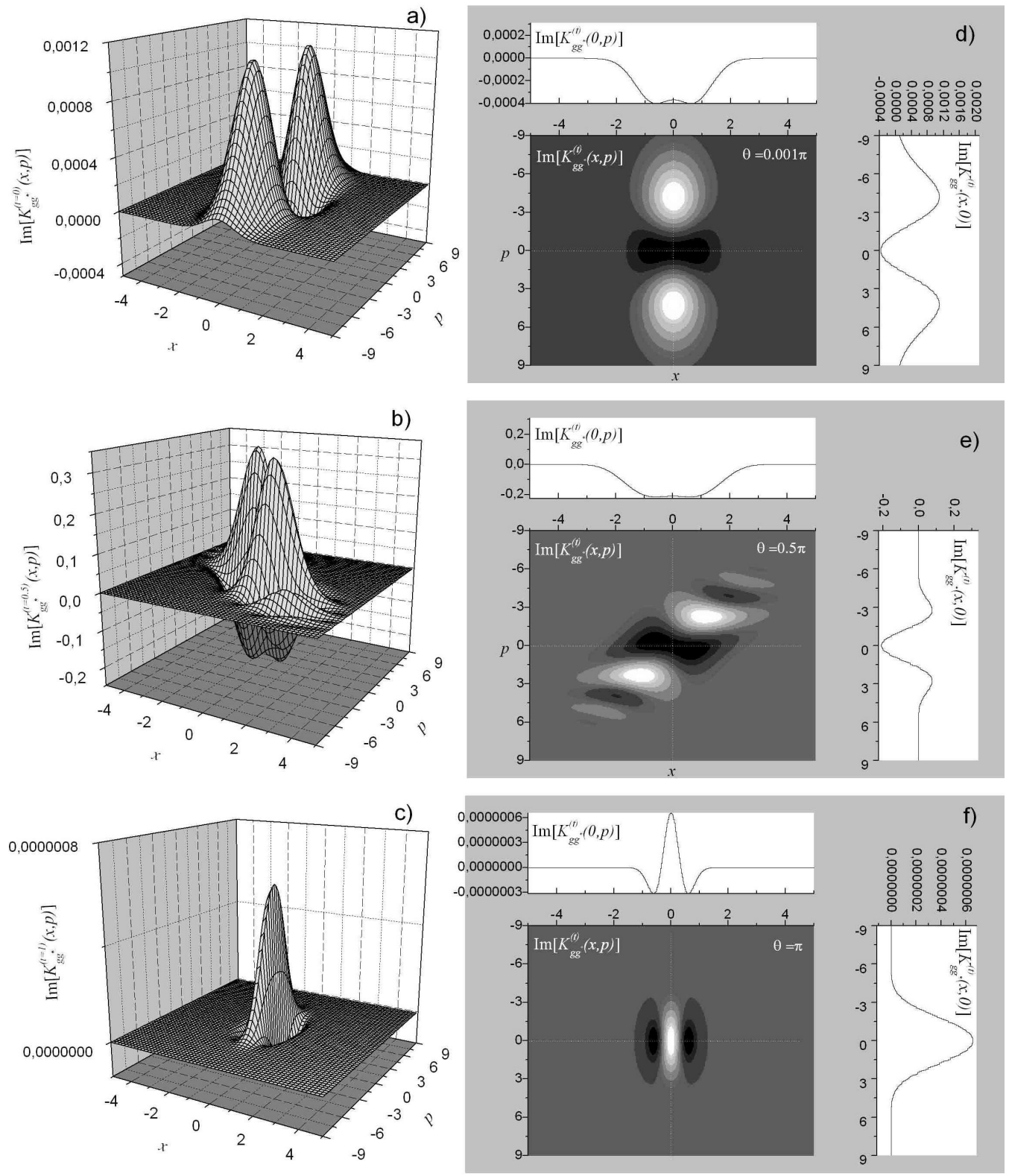

Fig. 2. Rotation of the joint distribution for the Gaussian signal (imaginary part) on the information diagram at different values of the joint parameter and $\sigma=0,5: t=0.001(\mathrm{a}, \mathrm{d}), t=0.5(\mathrm{~b}, \mathrm{e})$ and $t=1(\mathrm{c}, \mathrm{f})$.

imaginary part would decrease with decreasing $\theta$ angle. Hence, the joint distribution for the Gaussian signal is in fact real and positively defined for the parameter values $t \leq 0.001$ ( or $\theta \leq 0.18^{0}$ ). The alternative limit occurs at the joint parameter $t=1$, which corresponds to the rotation angle $\theta=180^{\circ}$. Unlike the previous case, this limiting transition may be exactly calculated. However, we should note the following. Since the expression (14) for the joint Gaussian distribution includes explicitly only the quantity 
$\theta / 2$, the practical rotation angle on the information diagram is equal to $\theta=90^{\circ}$. In this specific limiting case the real part of the joint distribution (see Fig. 1c, f) coincides exactly with the Wigner distribution calculated according to Eq. (9), whereas the imaginary part (see Fig. 2c, f) has the value of the order of $10^{-7}$, i.e. tends to zero, like in the previous limiting case. Thus, the joint distribution of the Gaussian signal is real and positive in the limiting case under interest.

Let us study the intermediate region between the above limiting cases, when we have the joint parameter $t=0.5\left(\theta=90^{\circ}\right)$. This case is specific since it describes a mixed state equidistant from the Wigner and Ville distributions. If $t=0.5$ we obtain the unit value of the other parameter, $T=1$. That is why the distribution formed in these conditions is rotated by the angle $\theta / 2=45^{\circ}$ with respect to both the Ville and Wigner distributions. The results calculated for the joint Gaussian distribution are shown in Fig. 1b, e (the real part) and Fig. 2b, e (the imaginary part). It is seen that the joint distribution is rotated by $\theta / 2=45^{\circ}$ and suffers a deformation. It is worth noticing that the rotations and deformations occur for the both real and imaginary parts of the joint distribution.

In other words, we arrive at the conclusion that the Wigner distribution of the Gaussian signal is formed as a result of rotation on the information diagram of the Ville distribution by the angle $\theta / 2=90^{\circ}$. Furthermore, availability of the $t$ parameter in the joint distribution allows investigating a continuous process of redistribution between the limiting distributions. While changing the $t$ parameter, we may study different mixed states of our system, in which the Wigner and Ville distributions are present in some definite proportions.

Now that we have already studied the region where the joint distribution is equidistant from the planes of Wigner and Ville distributions, let us pass to the regions of the joint distribution located close to one of those planes. On the initial stage of this analysis we should study the real and imaginary parts of the complex constant $C_{t t}(\theta)$ (see Eq. (15)), which governs the distribution amplitude.

The results of calculations of the complex parameter $C_{t t}(\theta)$ for different $\sigma$ values are depicted in Fig. 3. The plane $\theta=0^{\circ}$ corresponds to the Ville distribution, the plane $\theta=180^{\circ}$ to the Wigner one and the planes located in between describe the common region. Here we consider two characteristic cases appearing with the values $\sigma=1$ and $\sigma=0.5$. As seen from Fig. 3a, the value of this constant corresponds to the known results in the limiting cases. Its real part differs by the factor four for the Wigner and Ville planes, while the imaginary part is zero on those planes. Of a particular interest is redistribution of the $C_{t t}(\theta)$ constant for intermediate $\theta$ values. Here we observe a principled difference from the Wigner and Ville regions. We refer to the region close to the Ville plane $(t=0-0.3)$ as the region of Ville distribution. Likewise, the region located close to the Wigner plane $(t=0.9-1)$ may be called as the region of 

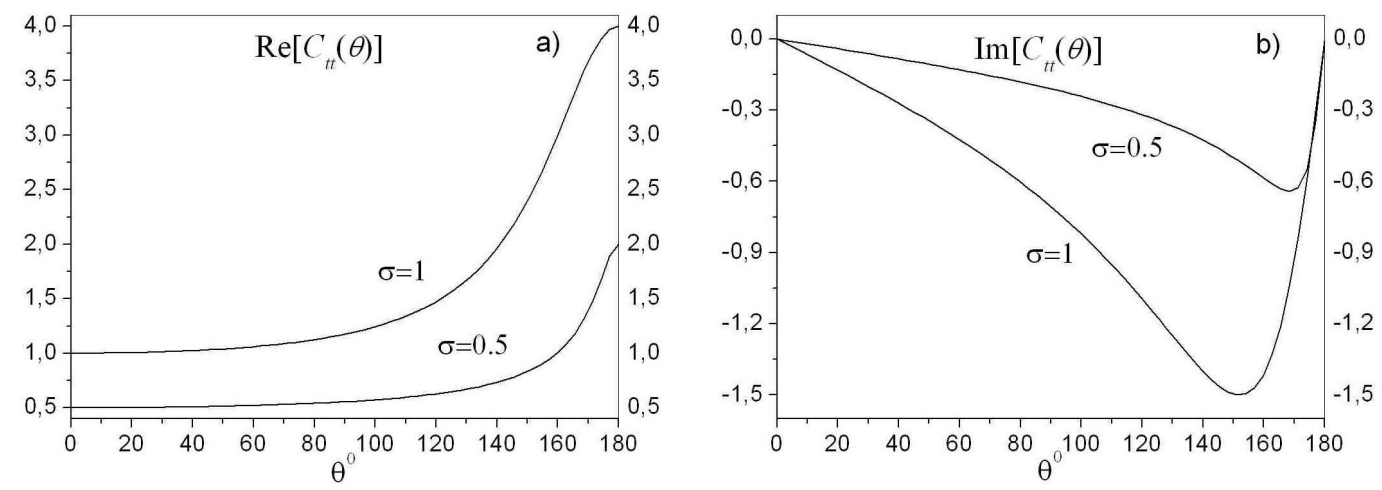

Fig. 3. Real and imaginary parts of the complex constant $C_{t t}(\theta)$ at different values of the $\sigma$ parameter.

Wigner distribution. In order to analyze the mentioned regions in a vivid manner, we will present the results calculated for the joint Gaussian distribution that correspond to those regions.

The Ville (Fig. 4) and Wigner (Fig. 5) distribution regions allow studying the formation of the joint distribution in a vicinity of the known limiting cases (i.e., the Ville and Wigner distributions). This would enable elucidating small deviations and changes in those distributions.

\section{Region of Ville distribution}

From Fig. 3 one can deduce the width of that region, i.e. the interval of $t$ values, for which the distribution under study would be visually similar to the Ville one. The
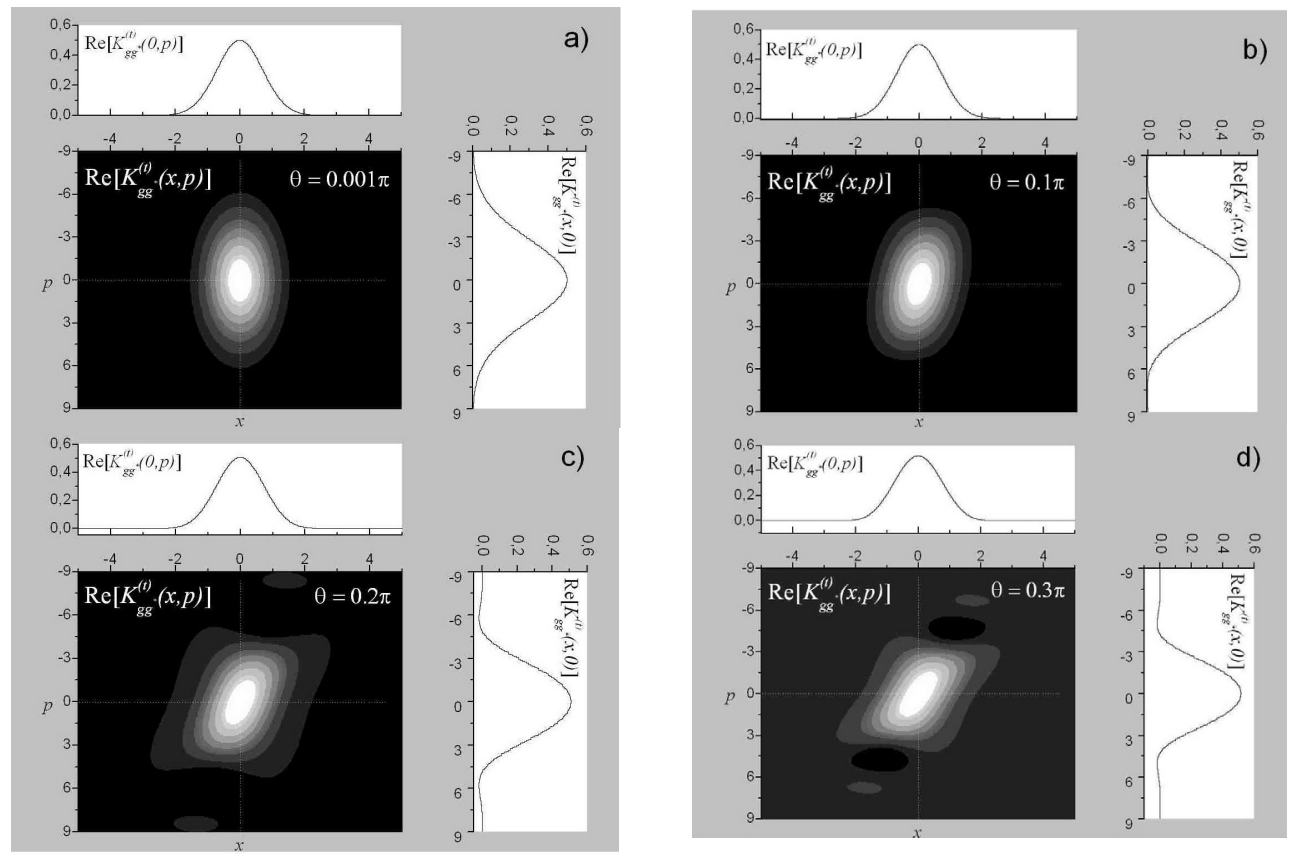

Fig. 4. Joint distribution of the Gaussian signal in the Ville domain (real part) at different values of the joint parameter.

Ukr. J. Phys. Opt. 2008, V9, №2 
corresponding width may be found when analyzing the curves depicted in Fig. 3. The characteristic region is located in the limits $t=0-0.3$. The results of formation of the joint distribution in that region are shown in Fig. 4. As demonstrated above, the Ville distribution is formed for the values $t \leq 0.001$ (Fig. 4a). When the joint parameter changes $(t=0.1-0.2-$ see Fig. $4 \mathrm{~b}, \mathrm{c})$, the joint distribution begins to rotate clockwise and simultaneously becomes deformed. It is worthwhile that the joint distribution for those cases still bears resemblance to the Ville distribution and its imaginary part remains comparatively small.

Further changes in the joint parameter ( $t=0.3-$ see Fig. $4 d)$ give rise to rotation by a larger angle $\left(\theta=54^{\circ}\right)$ and essential deformation. The joint distribution in this case has visually nothing in common with the Ville distribution.
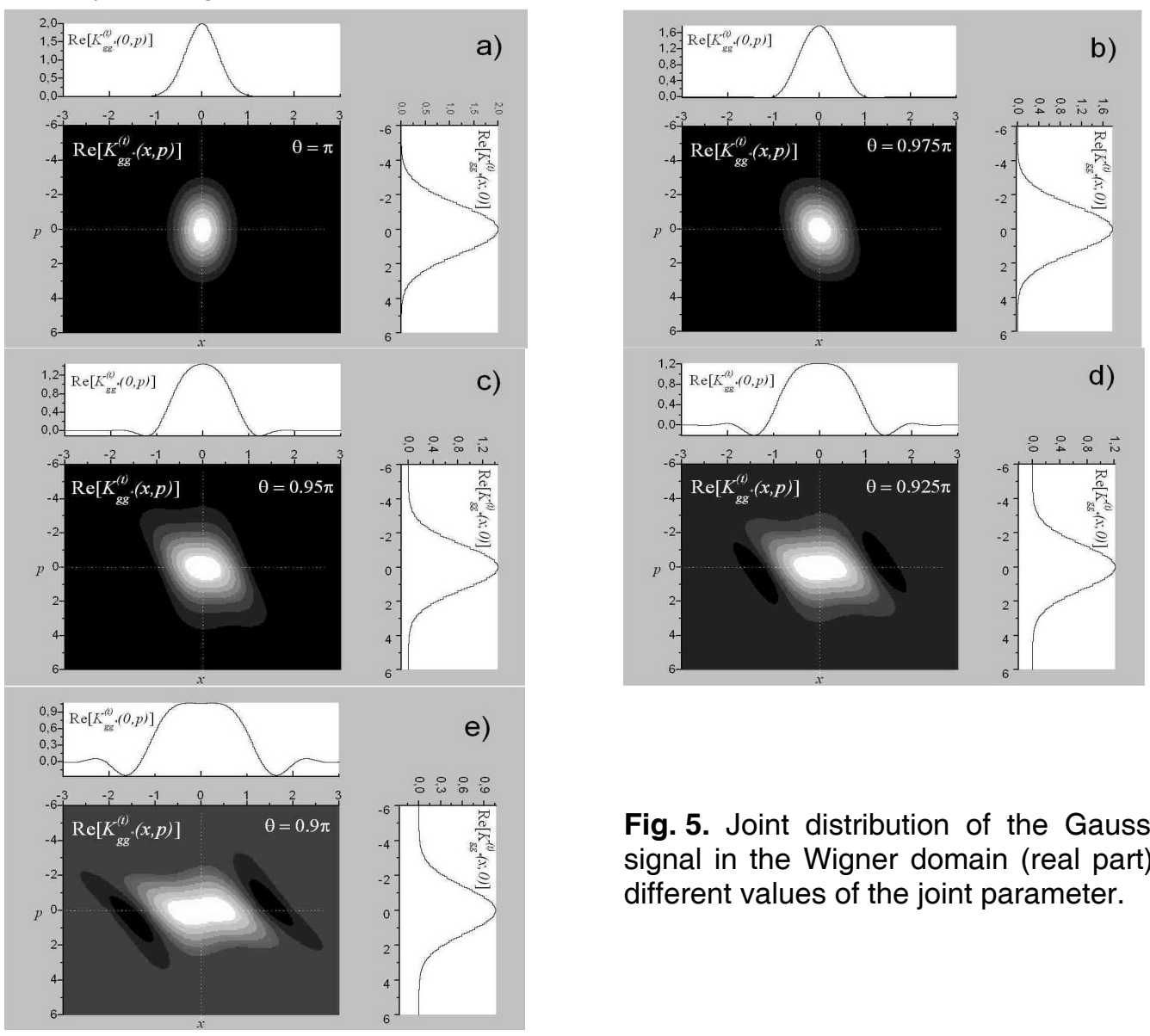

Fig. 5. Joint distribution of the Gaussian signal in the Wigner domain (real part) at different values of the joint parameter.

\section{Region of Wigner distribution}

According to Fig. 3, this region has much narrower limits in terms of the joint parameter $(t=0.9-1)$. This is caused by sharp changes in the real and imaginary parts of the $C_{t t}(\theta)$ constant within the region. Similar to the previous case, the region of the Wigner distribution is formed close to the limit $t=1$ (see Fig. 5a). With small changes of the 
joint parameter $(t=0.975-0.95$ - see Fig. 5b, c) the joint distribution rotates anticlockwise and simultaneously changes its form. Here the resulting distribution resembles the Wigner one. At further rotation, transformation of the distribution makes its visual identification with the Wigner distribution impossible (see Fig. 5d, e).

There is a possibility for studying the properties of the Wigner and Ville distributions appearing at small deviations of the joint parameter, with the aim of analyzing redistribution mechanism and the appropriate applications for description of optical systems. The Wigner and Ville distributions move towards each other on the information diagram and 'intersect' at the point $t=0.5$ (see Fig. 1b, e and Fig. 2b, e). With further rotation, the Ville distribution degenerates into the Wigner one, and vice versa.

\section{Conclusions}

In this work we propose a joint spatial-frequency distribution as generalization of the two known distributions suggested by Wigner and Ville. The main characteristic feature of our study is that, contrary to the other earlier generalizations, our distribution permits tracing not only the limiting cases but a continuous transition among different intermediate distributions. This is due to introduction of the joint parameter $t$, of which changes lead to different distributions. Thus, beside of the Wigner $(t=1)$ and the Ville ( $t=0)$ distributions, there exist a whole set of other distributions $(0<t<1)$, which describe some 'mixed' states located between the limiting cases. A similar distribution has been introduced by S. Chountasis [13] basing on the Wigner formalism. We introduce the joint distribution on the basis of Ville formalism, thus suggesting a number of advantages associated with reconstructing signal intensity following from the distribution [12, 16]. As mentioned in [13], distributions of such a type may be considered in a general context of Wigner tomography.

Analytical formulae are derived for the joint distribution concerned with the Gaussian signal and their graphic representations are discussed. Formation of the Gaussian signal is investigated in detail for the common region and different values of the $t$ parameter. It is revealed that rotation of the Ville distribution on the information diagram occurs with changing $t$ parameter, with the rotation angle equal to $\theta=t \pi$. It is also demonstrated that transition between the Wigner and Ville distributions takes place through the complex plane.

The joint distribution suggested by us represents a valuable addition to the Wigner and Ville distributions. It is shown that the joint distribution can be treated as quasi-Ville one in the parameter region $t \in[0,0.3]$ or quasi-Wigner distribution for the values of $t \in[0.9,1]$.

\section{References}

1. Cohen L, 1989. Time-frequency distributions - A review. IEEE. 77: 941-981.

2. Boashash B., Time-frequency Signal Analysis and Processing, Elsevier, (2003).

Ukr. J. Phys. Opt. 2008, V9, №2 
3. Cristobal G, Gonzalo C, Bescos J, 1991. Image filtering and analysis through the Wigner distribution. Advances in Electronics and Electron Physics Series 80: 309397.

4. Cohen L., Time-frequency Analysis, Prentice Hall, (1995).

5. Mertings A., Signal Analysis, Wiley\&Sons, (1999).

6. Kurtsiefer Ch, Pfau T, Mlynek J. 1997. Measurement of the Wigner function of an ensemble of helium atom. Nature. 386: 150-153.

7. Breitenbach G, Schiller S, Mlynek J, 1997. Measurement of the quantum statesof squeezed light. Nature. 387: 471-475.

8. Smithey D, Beck M, Raymer M, 1996. Measurement of Wigner distribution and the Density Matrix of a Light Mode Using Optical Homodyne tomography: Application to Squeezed States and the Vacuum. Phys. Rev. Lett. 70: 1244-1247.

9. Cohen L, 1966. Generalized phase-space distribution functions. J.Math.Phys. 7: 781786.

10. De Bruijn N, 1973. A theory of generalized functions, with applications to Wigner distribution and Weyl correspondence. Nieuw Archief voor Wiskunde. 3(XXI): 205-280.

11. Durak L. Novel time-frequency analysis technique for deterministic signals, $\mathrm{PhD}$ thesis, Institute of engineering and science of Bilken University. Turkey, (2003).

12. Shovgenyuk MV, Kozlovskii YuM, 2000. Similitary of conjugate images at fractional Fourier transform. Dop. NAN Ukraine 6: 92-97.

13. Chountasis S, Vourdas A and Bendjaballah C, 1999. Fractional Fourier operators and generalized Wigner functions. Phys. Rev. A. 60: 3467-3473.

14. Chountasis S, Vourdas A, 1998. Weyl functions and their use in the studyof quantum interference. Phys. Rev. A. 58: 848-855.

15. Chountasis S, Vourdas A, 1998. Weyl and Wigner functions in an extended phasespace formalism. Phys. Rev. A. 58: 1794-1798.

16. Shovgenyuk MV, Preprint ICMP-92-25U, Lviv, (1992).

17. Kozlovskii YuM 2003. Generalized fractional Fourier transform and optical systems. Ukr.J.Phys.Opt. 3: 124-134. 